\title{
A Review on Detection and Diagnosis of Osteoporosis
}

\author{
Soubarnika $\mathbf{S}^{1}$, Prof. P.Nandakumar ${ }^{2}$ \\ PG Scholar, ECE Department, NSS College of Engineering, Palakkad, Kerala, India ${ }^{1}$ \\ Professor, ECE Department, NSS College of Engineering, Palakkad, Kerala, India ${ }^{2}$
}

\begin{abstract}
Osteoporosis is a kind of a skeletal system disease mainly categorized by alterations in bone mass density and its structure that makes the bone prone to fracture. The assessment of osteoporotic disease from bone radiograph images presents a major challenge for pattern recognition. Textured images of osteoporotic and healthy subjects show high degree of resemblance, it increases the difficulties in discriminating such textures. Early diagnosis of this disease is vital for a healthy living condition.
\end{abstract}

Keywords: Bone mineral density, artificial neural network, Auto encoder, Osteoporosis, SVM classifier

\section{INTRODUCTION}

Osteoporosis affects over 3 million people in UK. More than 500,000 people receive hospital treatment for fragility fractures every year as a result of osteoporosis. Osteoporosis is a disease in which bone weakening increases the risk of a broken bone [1].Characterization of Bone radio- graph images is a task in the osteoporosis diagnosis. Osteoporosis is mainly categorized by decrease in the bone mass and structure. It increases the hazard of fracture. It is difficult to identify the normal subjects from that of osteoporotic because they show high degree of resemblance in their texture. It occurs most commonly in women because of hormonal changes (menopause).Generally occurs mainly in spine, hips and wrist. By knowing the quality of the bone, one can prevent the fracture risk [11]. Clinically, to diagnose osteoporosis the classical method consists in measuring the bone mineral density (BMD) and compares it with that of a healthy young adult. The results of this test are expressed in T-score. A bone density with a $\mathrm{T}-$ score $\geq-1$ is classified as a normal. While a $\mathrm{T}-$ score $\leq-2.5$ indicates that the bone has osteoporosis [2].

In this paper several methods are studied and analysed for detecting and diagnosing osteoporosis using bone mineral density including artificial neural network (ANN), x-ray imaging technique, stacked sparse auto encoder and fuzzy neural network.

\section{METHODOLOGY}

In [2], Stacked Sparse Auto Encoder (SSAE) is a deep learning architecture of sparse autoencoders consisting of a series of sparse autoencoder networks, stacked above each other in which the outputs of each hidden layer of an autoencoder are wired to the input layer of the successive network. An auto-encoder is a neural network that aims to minimize the reconstruction error between the input data $x$ and the output data ${ }^{\wedge} \mathrm{x}$ to learn the high-level features.

SSEA is training by applying the greedy layer-wise training strategy in which way each autoencoder tries to minimize the reconstruction errors of the previous layer. To sum up, SSAE is applied to learn the high-level features from original input. Features are learned by optimizing the cost function and are encapsulated in weights.

To classify an image from it pixels, our model performs a series of three consecutive steps: A) preprocessing, B) image subdivision and feature extraction, C) pooling and classification.

A comparison study on the performance of different SVM kernel functions (i.e. Linear, Polynomial, RBF and Sigmoid) for classification was performed. The SVM aims to achieve good separation between classes by finding a set of hyper planes that maximizes the functional margin, where the distance to the nearest training example is the greatest. It is shown that the obtained rates demonstrate that the accuracies of Linear SVM classifier (95.50\%) are better than the other kernel functions. Once the deep network learns the local features, the signatures of training images are constructed by pooling the features extracted from the patches belong to the same image. These signatures are associated with its label inorder to training the SVM classifier. SVM are employed to classify these signatures in order to distinguish between the Osteoporosis Population (OP) and Control Cases (CC). The introduction of deep learning for the diagnosis of osteoporosis has given promising results with an accuracy rate around $95.5 \%$. 


\section{International Advanced Research Journal in Science, Engineering and Technology}

Vol. 6, Issue 5, May 2019

In [5], a novel learning-driven system Intelligent Medical Diagnostic System (IMDS) accessible through web interface for performing on-line initial screening for osteoporosis. The fundamentals of the IMDS are mainly based on the wellknown theory of Fuzzy-Neural Networks (FNNs). FNNs possess the ability to automatically learn useful knowledge from available examples without the active participation of domain experts during the process of constructing system, and can exhibit superiority over other conventional technologies (e.g. statistical techniques and regression analyses) due to its superior nonlinear modelling. It is well known that FNNs possess the ability to automatically acquire useful knowledge from a large number of examples, and can exhibit superior nonlinear modelling capability in many fields such as pattern classification, object recognition, model prediction, and system identification. A FNN is named by the fact that a fuzzy model described by if-then rules and inference mechanisms can completely be transformed into a Multi- Layered Artificial Neural Network (MNN) composed of neurons and connecting links as long as each neuron in a MNN performs a corresponding function in a fuzzy model. This fact makes standard gradient learning methods in MNNs available to adjust the parameters of FNNs; the proposed FNN consists of the following four layers in sequential order: an input layer, a fuzzification layer, a rule layer, and a class layer.

The IMDS (Intelligent Medical Diagnostic System) system is mainly comprised of three parts: a learning module, a knowledge base, and a web-based user interface. The objective of the learning module is to construct a FNN classifier not only with high classification accuracy but also with good interpretability. This objective is accomplished by means of a series of learning algorithms including k-means clustering, gradient descent parameter tuning, and similarity-based model simplification. The knowledge base is practically a FNN classifier. It consists of a fuzzy inference engine and a fuzzy medical rule database. The former performs fuzzy inference with respect to the presented input feature vectors so as to provide reasonable diagnostic results; the latter contains a collection of diagnostic rules acquired from available medical databases which store a great amount of data relating to patient's records or medical history. The web-based user interface provides users with a convenience path to access the service offered by the IMDS. Through this interface, users can submit their personal information and receive the related diagnoses in an easy manner.

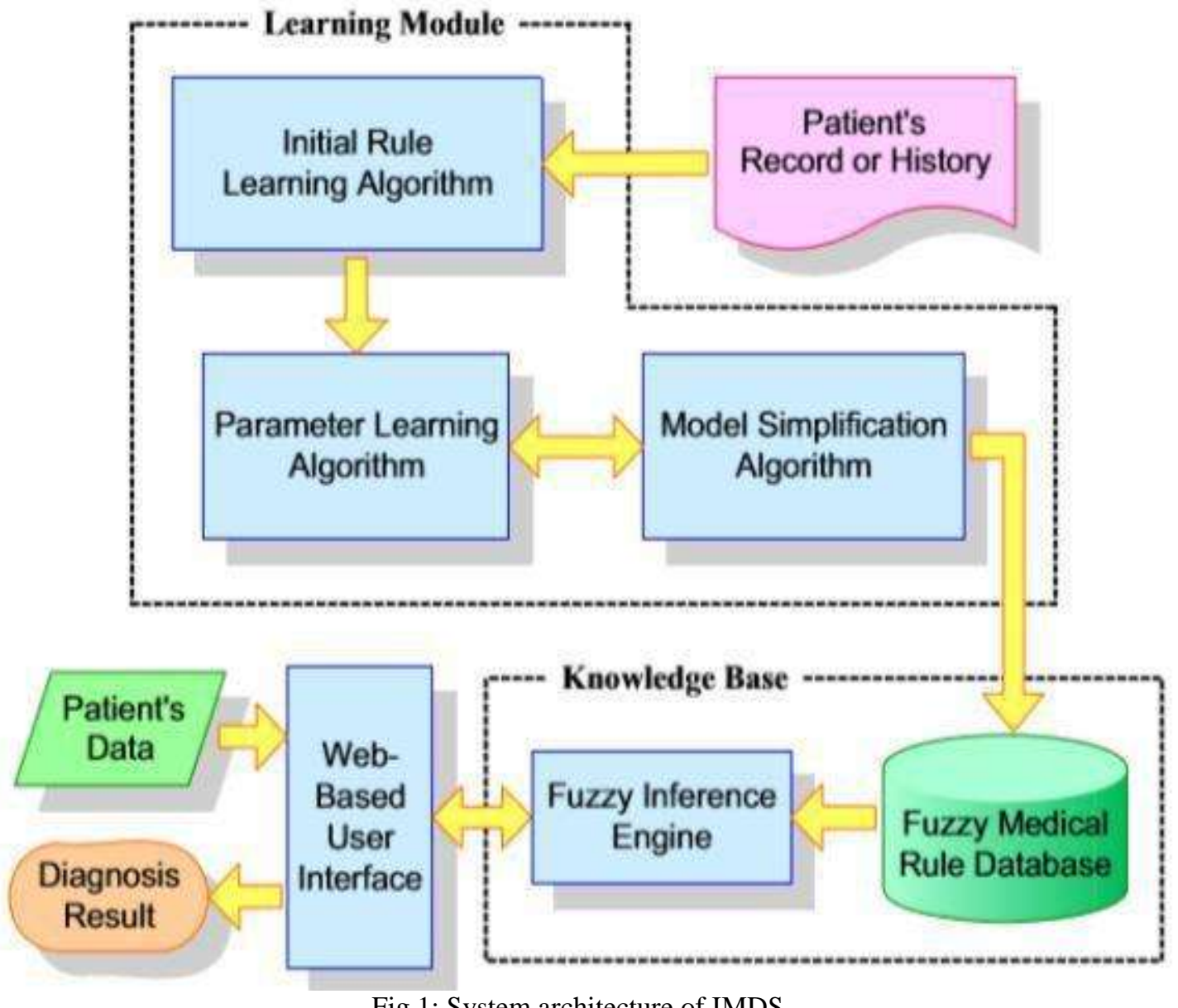

Fig 1: System architecture of IMDS

Advantages of the IMDS are four-fold: (1) automatically generating diagnostic rules without medical experts' active participation can significantly reduce the construction cost of the system; (2) the learning-from-example ability of the 


\section{International Advanced Research Journal in Science, Engineering and Technology}

Vol. 6, Issue 5, May 2019

IMDS can extract implicit, previously unknown, and potentially useful medical knowledge from considerable patient's data or medical history; (3) using a questionnaire of osteoporosis risk factors rather than other invasive methods or laboratory measurements to assess osteoporosis risk not only can considerably reduce the cost of mass screening, but can also speed up screening process; and (4) the convenient, interactive, web-based user interface can effectively increase the accessibility of the system. Moreover, it should be noted that the reliability of the system mainly depends on the information supplied by the users.

In [8], to achieve a diagnosis system of osteoporosis with the assistance of a network, an artificial neural network model is established and applied. Then parts of patients are selected randomly as the training set and the rest is regarded as the prediction set. Input score results and bio chemical parameters are related to osteoporosis. The prediction results of all samples are compared with the predicted results of logistic regression. Diagnostic results of the artificial neural network model are compared with the results of logistic regression.

Artificial Neural Network (ANN) is the abstraction and simulation of a number of basic features extracted from neurophysiological models. Its organizations can simulate the interaction which is made in response to objects in the real world by biological nervous system. This is a basic processing unit of the neural network, connects with each other and accepts a weighted input to produce a corresponding output by means of an activation function. In the neural network, the ability and efficiency to solve the problem, to a large extent, depends on the activation function used by the network in addition to the network structure. The choice of the activation function has great influence on the convergence speed of the network. The choice of activation function should be different according to different actual problem. In this paper, we adopt the logarithmic sigmoid function which is the most widely used activation function in neurons. In the process of learning and training, weights and thresholds change constantly. Different output is decided by different weights and thresholds. The appropriate network and the function approximation will be achieved. The Back-Propagation algorithm is a multilayer feed-forward ANN and is commonly adopted for solving classification problems. It is considered to be a kind of learning method because it can constantly amend the connection weights between different artificial neurons which can make up forward multi-layer network. So that the forward multi-layer network is able to change the input information into the desired output information. It is referred to as backward learning algorithm because when the connection weights of artificial neurons are modified based on the difference, which will be reversed layers one by one to the back propagation, between the actual output and its desired output of the network to determine the modifications of connection weights.

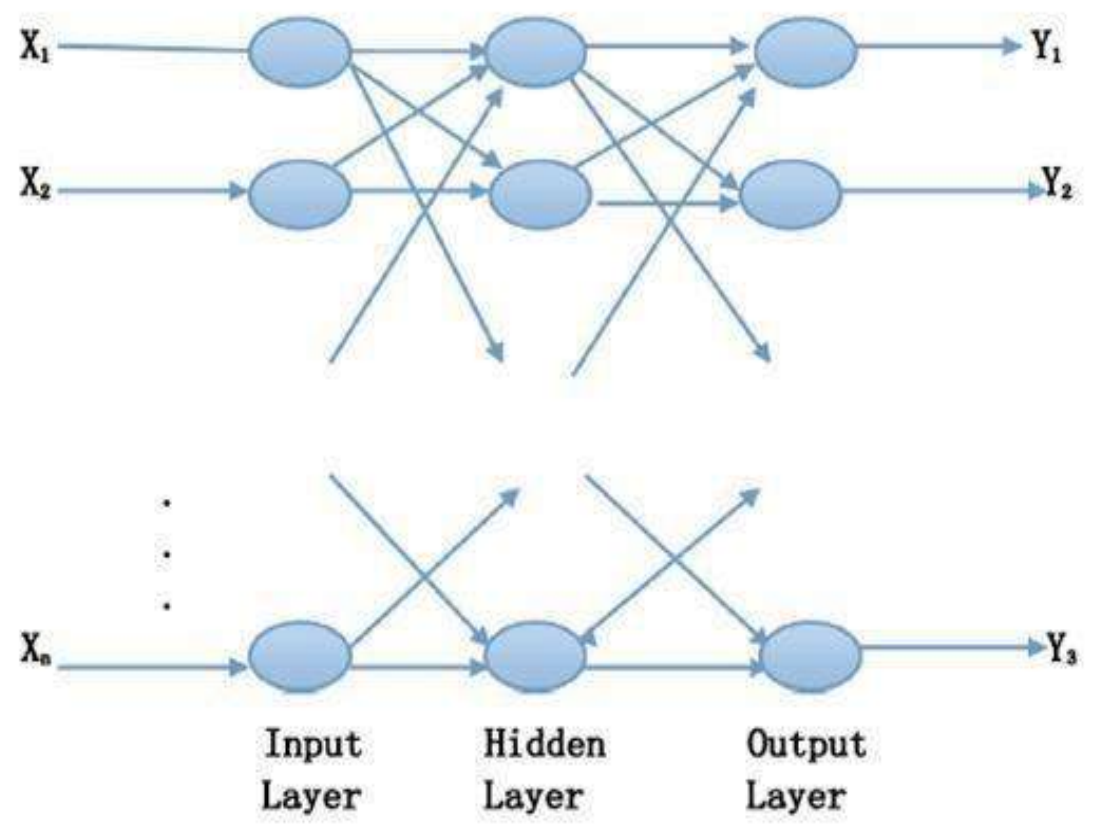

Fig 2: Back Propagation network structure

In this study, there are still some limitations. On the one hand, the number of selected cases is a bit small and consequent, the network is not steady enough. Even though satisfactory results were obtained, till the neural networks were repeatedly trained several times. Although there are aforementioned limitations, the conducted study provides an alternative approach to diagnose osteoporosis. 


\title{
International Advanced Research Journal in Science, Engineering and Technology
}

\author{
Vol. 6, Issue 5, May 2019
}

\section{CONCLUSION}

Several techniques and methods in the diagnosis of osteoporosis were presented. In order to improve diagnostic accuracy and automation of this task, several methods uses texture analysis to extract features from bone X-ray images. Among these methods, SSAE to extract high-level feature from pixel intensities in an unsupervised manner. Stacked encoder uses pooling operation to construct image signatures from the high-level features extracted in the previous step and employ SVM to classify these signatures. The introduction of deep learning for the diagnosis of osteoporosis has given promising results with an accuracy rate around 95.5\% Intelligent Fuzzy-Neural Diagnostic System is convenient, interactive, web-based user interface can effectively increase the accessibility of the system. Reliability of the system mainly depends on the information supplied by the users. In the next method, ANN and logistic regression have been compared in order to explore an optimal method to diagnose osteoporosis.

\section{REFERENCES}

[1]. https://en.wikipedia.org/wiki/Osteoporosis

[2]. Yassine Nasser1, Mohammed El Hassouni1, "Diagnosis of osteoporosis disease from bone X-ray images with Stacked Sparse Autoencoder and SVM classifier", International Conference on Advanced Technologies for Signal and Image Processing - ATSIP'2017.

[3]. Kanis, J. A., Melton, L. J., Christiansen, C., Johnston, C. C., \& Khaltaev, N ,’The diagnosis of osteoporosis", Journal of bone and mineral research, 9(8), 1137-1141.

[4]. Tafraouti, A., El Hassouni, M.,Toumi, H., Lespessailles, E., \& Jennane, "Osteoporosis diagnosis using steerable pyramid decomposition \& fractional Brownian motion", in Image Processing Theory, Tools \& Applications(IPTA), 2015 International Conference on (pp. 309-312). IEEE.

[5]. Chin-Ming Hong, Chin-Teng Lin," An Intelligent Fuzzy-Neural Diagnostic System for Osteoporosis Risk Assessment", World Academy of Science, Engineering and Technology International Journal of Health and Medical Engineering Vol:2, No:6, 2008.

[6]. World Health Organization, "Assessment of fracture risk and its application to screening for postmenopausal osteoporosis," WHO, Geneva, Report series 843, 1994.

[7]. K. Polat, and S. Günes, "An expert system approach based on principal component analysis and adaptive neuro-fuzzy inference system to diagnosis of diabetes disease," Digit. Sign. Proc., vol. 17, no. 4, pp. 702-710, 2007

[8]. C. M. Hong, C. M. Chen, S. Y. Chen, and C. Y. Huang, "A novel and efficient neuro fuzzy classifier for medical diagnosis," Inter. Joint Conf. on Neur. Net., IJCNN'06, pp. 735-741, 2006.

[8]. Xinghu Yu a, ChaoYe," Application of artificial neural network in the diagnostic system of osteoporosis", Science Direct Neural Computing 2016.

[9]. H.R. Karimi,H.Gao, "New delay-dependent exponential synchronization for uncertain neural networks with mixed time delays, IEEE Trans.Syst.,Man., Cybern, 2010.

[10]. H. Fei, De-Shuang Huang, "A new constrained learning algorithm for function approximation by encoding apriori information into feed forward neural networks", NeuralComput.Appl.17(2008)433-439.

[11]. Kavya R, Dr.Joshi Manisha,'Texture based bone radiograph image analysis for Osteoporosis",IJERT 2015

[12]. J.Qiu,S.Ding,H.Gao,S.Yin, "Fuzzy-model based reliable static output feed-back H- infinity control of nonlinear hyperbolic PDE systems", IEEE Trans. Fuzzy Syst. (2015). 